\title{
Green-synthesized CdS nano-pesticides: toxicity on young instars of malaria vectors and impact on enzymatic activities of the non-target mud crab Scylla serrata
}

\begin{abstract}
Currently, nano-formulated mosquito larvicides have been widely proposed to control young instars of malaria vector populations. However, the fate of nanoparticles in the aquatic environment is scarcely known, with special reference to the impact of nanoparticles on enzymatic activity of non-target aquatic invertebrates. In this study, we synthesized CdS nanoparticles using a green protocol relying on the cheap extract of Valoniopsis pachynema algae. CdS nanoparticles showed high toxicity on young instars of the malaria vectors Anopheles stephensiand $A$. sundaicus. The antimalarial activity of the nanosynthesized product against chloroquine-resistant (CQ-r) Plasmodium falciparumparasites was investigated. From a non-target perspective, we focused on the impact of this novel nano-pesticide on antioxidant enzymes acetylcholinesterase (AChE) and glutathione Stransferase (GST) activities of the mud crab Scylla serrata. The characterization of nanomaterials was carried out by UV-vis and FTIR spectroscopy, as well as SEM and XRD analyses. In mosquitocidal assays, $\mathrm{LC}_{50}$ of $V$. pachynema-synthesized $\mathrm{CdS}$ nanoparticles on A. stephensi ranged from 16.856 (larva I), to $30.301 \mu \mathrm{g} / \mathrm{ml}$ (pupa), while for $A n$. sundaicus they ranged from 13.584 to $22.496 \mu \mathrm{g} / \mathrm{ml}$. The antiplasmodial activity of $V$. pachynema extract and CdS nanoparticles was evaluated against CQ-r and CQ-sensitive (CQs) strains of Plasmodium falciparum. $\mathrm{IC}_{50}$ of $V$. pachynema extract was $58.1 \mu \mathrm{g} / \mathrm{ml}$ (CQ-s) and $71.46 \mu \mathrm{g} / \mathrm{ml}$ (CQ-r), while nano-CdS IC 50 was $76.14 \mu \mathrm{g} / \mathrm{ml}$ (CQ-s) and $89.21 \mu \mathrm{g} / \mathrm{ml}$ (CQr). In enzymatic assays, $S$. serrata crabs were exposed to sub-lethal concentrations, i.e. 4, 6 and $8 \mu \mathrm{g} / \mathrm{ml}$ of $\mathrm{CdS}$ nanoparticles, assessing changes in GST and AChE activity after 16 days. We observed significantly higher activity of GST, if compared to the control, during the whole experiment period. In addition, a single treatment with $\mathrm{CdS}$ nanoparticles led to a significant decrease in AChE activity over time. The toxicity of CdS nanoparticles and Cd ions in aqueous solution was also assessed in mud crabs, showing higher toxicity of aqueous $\mathrm{Cd}$ ions if compared to nano-CdS. Overall, our results underlined the efficacy of greensynthesized CdS nanoparticles in malaria vector control, outlining also significant impacts on the enzymatic activity of non-target aquatic organisms, with special reference to mud crabs.
\end{abstract}

Keyword: Acetylcholinesterase; Anopheles stephensi; Biosafety; Glutathione S-transferase; Malaria vector; Nanosynthesis 